\title{
Revealing exotic magnetic states through machine learning assisted modeling of neutron diffuse scattering data \\ David Tennant ${ }^{1}$ \\ ${ }^{1}$ Neutron Sciences Directorate, ORNL tennantda@ornl.gov
}

\begin{abstract}
Neutron scattering has a number of distinctive features. It is isotope sensitive, and readily detects light elements, as well as having a magnetic scattering cross section. The scattering formulae are relatively simple but measurements involve large amounts of noisy data. The data includes background and artifacts that are difficult to handle and in the case of diffuse and inelastic scattering 3D and 4D data that are challenging to visualize. Further, there is no solution to the inverse scattering problem. Experiments conventionally are limited by the lack of theory to interpret the scattering making it hard to optimally perform experiments and analyze results. Data science approaches are needed to solve these challenges. Computational simulations on wide ranges of cases provide the fundamental knowledge to interpret and guide the experiments. Integration of machine learning into the neutron scattering pipeline provides a way to handle the large numbers of simulations and volume of data and integrate these together. We have recently demonstrated integration of machine learning into neutron scattering for diffuse and i32rnelastic scattering. These involve 3D and 4D data sets and require sophisticated modeling. These are providing new insights into exotic magnetic states and I will show two examples of frustrated magnetic materials where we have applied these new approaches allowing the solution of difficult science problems.
\end{abstract}

